\title{
Hamster Cholangiocarcinoma
}

National Cancer Institute

\section{Source}

National Cancer Institute. Hamster Cholangiocarcinoma. NCI Thesaurus. Code C134566.

Cholangiocarcinoma that occurs in a hamster. 